\title{
Postoperative hiatal hernia after minimally invasive esophagectomy for esophageal cancer
}

\author{
Hironori Iwasaki $^{1,2}$, Tomokazu Tanaka ${ }^{2}$, Shuusuke Miyake ${ }^{2,3}$, Yukie Yoda ${ }^{2}$, Hirokazu Noshiro ${ }^{2}$ \\ ${ }^{1}$ Department of Surgery, Japanese Red Cross Kumamoto Hospital, Kumamoto, Japan; ${ }^{2}$ Department of Surgery, Saga University Hospital, Saga, \\ Japan; ${ }^{3}$ Department of Surgery, Takagi Hospital, Fukuoka, Japan \\ Contributions: (I) Conception and design: H Iwasaki, Y Yoda, H Noshiro; (II) Administrative support: H Iwasaki, T Tanaka, Y Yoda, H Noshiro; (III) \\ Provision of study materials or patients: H Iwasaki, T Tanaka, S Miyake, H Noshiro; (IV) Collection and assembly of data: H Iwasaki, S Miyake; \\ (V) Data analysis and interpretation: H Iwasaki, T Tanaka, S Miyake, H Noshiro; (VI) Manuscript writing: All authors; (VII) Final approval of \\ manuscript: All authors. \\ Correspondence to: Hironori Iwasaki, MD, PhD. Department of Surgery, Japanese Red Cross Kumamoto Hospital, 2-1-1 Nagamine-minami, Higashi- \\ ku, Kumamoto 861-8520, Japan. Email: hiwasaki0519@gmail.com.
}

Background: Minimally invasive esophagectomy (MIE) can reduce various complications compared with conventional thoracotomic esophagectomy. However, several reports suggested that MIE promoted incidence of post-operative hiatal hernia $(\mathrm{HH})$. In current reports, we retrospectively analyzed incidence and risk factors of $\mathrm{HH}$ development after MIE.

Methods: A total of 113 patients undergoing MIE (McKeown esophagectomy) at our institute from April 2009 to December 2015 were included in this study. Patients with clinical stage II and III received neoadjuvant chemotherapy (NAC).

Results: Eleven of 113 patients (9.7\%) undergoing MIE developed HH. Four of them were female and the ratio of female among the patient with $\mathrm{HH}$ was higher than that among the patient without $\mathrm{HH}$ after MIE (36.4\% vs. $13.7 \%, \mathrm{P}=0.05$ ). Sixty-six patients (58.4\%) during the study period were administered NAC and 10 of 11 patients with $\mathrm{HH}(90.9 \%)$ received NAC according to the clinical stage, which was significantly more than in the non-HH group $(\mathrm{P}=0.02)$. Type and route of graft organ were not related to $\mathrm{HH}$ development. Moreover, the fixation of the conduit organ at the hiatus does not contribute to post-operative $\mathrm{HH}$.

Conclusions: In the current study, we showed that NAC was a major risk factor of $\mathrm{HH}$ development after MIE.

Keywords: Complications; esophageal cancer; hiatal hernia (HH); minimally invasive esophagectomy (MIE); neoadjuvant chemotherapy (NAC)

Submitted Mar 16, 2020. Accepted for publication Aug 06, 2020.

doi: $10.21037 /$ jtd-20-1335

View this article at: http://dx.doi.org/10.21037/jtd-20-1335

\section{Introduction}

Esophageal cancer is one of the most aggressive diseases, and its incidence is rapidly increasing worldwide (1). Both the mortality and morbidity are still high compared with those of other gastrointestinal cancers (2). However, advances in the early detection and multimodal therapies have improved the survival of the patients $(3,4)$. According to a comprehensive registry of esophageal cancer in Japan (5), the 5 -year overall survival rate is $55.5 \%$. As the overall survival period after esophagectomy is getting longer, complaints should be surveyed more carefully in order to ensure an adequate quality of life in patients who undergo esophagectomy. In order to reduce the surgical invasiveness and thereby improve the postoperative quality of life, thoracoscopic and/or laparoscopic surgery for 
esophageal cancer was introduced by Cuschieri et al. in the early 1990s (6). The use of minimally invasive surgery (MIS) can reduce the pain and systemic inflammatory response. Minimally invasive esophagectomy (MIE) can also reduce the duration of the postoperative stay and pain as well as the respiratory complications compared with conventional thoracotomic esophagectomy (7).

However, several drawbacks to MIE have also been recognized. Postoperative hiatal hernia $(\mathrm{HH})$ is a wellknown complication after conventional thoracotomic esophagectomy, and MIE promotes its incidence, likely due to the paucity of adhesions in MIS (8-10). While MIE is becoming widely performed, the risk of $\mathrm{HH}$ development after MIE has not been well documented.

In this study, we determined the incidence of postoperative $\mathrm{HH}$ after MIE and analyzed the risk factors using a prospectively maintained comprehensive surgical database at our institute. We present the following article in accordance with the STROBE reporting checklist (available at http://dx. doi. org/10.21037/jtd-20-1335).

\section{Methods}

\section{Patients}

All patients who had undergone esophagectomy for esophageal cancer as a part of a three-stage procedure (McKeown esophagectomy) at our institute from April 2009 to December 2015 were reviewed and enrolled in this study. During this period, all patients were treated by MIE. MIE was introduced in April 2009 by a senior surgeon who had had experience treating over 180 cases by MIE at another hospital. This senior surgeon operated on or supervised all patients at our hospital. According to the guideline published by the Japanese Esophageal Society $(4,11)$, patients with clinical stage II or III disease received neoadjuvant chemotherapy (NAC) with a regimen of 5 -fluorouracil and cisplatin (FP) or modified docetaxel, cisplatin and 5-fluorouracil (DCF). In addition, patients with suspicious $\mathrm{T} 4$ tumors or bulky nodal involvement received preoperative radiation therapy.

Complications accompanying esophagectomy were prospectively checked in all patients during admission and after leaving the hospital. Standard surveillance of the patients, including chest X-ray and computed tomography (CT), was carried out every three to 6 months for at least 5 years. Upper gastrointestinal endoscopy was performed every year. All patients with $\mathrm{HH}$, regardless of symptoms, underwent laparoscopic repair at our hospital.

The study was conducted in accordance with the Declaration of Helsinki (as revised in 2013). We obtained informed consent from all of the patients enrolled in this study, and this retrospective study was approved by the Institutional Review Board of Saga University Hospital (2017-04-22).

\section{MIE}

All patients in this study were treated by thoracoscopic or robot-assisted thoracic procedures in the prone position, as described in our previous reports (12-14). The extent of lymph node dissection in the thoracic and abdominal procedures did not differ markedly by stage. In patients whose stomach was available as a conduit, the stomach was mobilized laparoscopically. Preservation of the left inferior phrenic artery was attempted in all patients. The lateral segment of liver was not mobilized. The esophageal hiatus of the diaphragm was enlarged vertically about $2-4 \mathrm{~cm}$ at the central tendon in order to pass the gastric conduit smoothly. The excised esophagus and the mobilized stomach were extracted thorough the $3-4 \mathrm{~cm}$ minilaparotomy at the umbilicus. A gastric conduit $4 \mathrm{~cm}$ in width was created extracorporeally. The gastric conduit was then pulled up through the posterior mediastinum if possible, and anastomosis was performed at the neck. The organ for reconstruction was then pulled-down transabdominally into the abdomen in order to straighten it and thereby avoid bending of the redundant conduit. Finally, the conduit organ was fixed at the diaphragm with a couple of stitches using absorbable or non-absorbable monofilament sutures to prevent postoperative $\mathrm{HH}$. As described later, some modifications were made to this fixation procedure during the study period.

In patients whose stomach was not available as a conduit due to previous or synchronous gastrectomy, reconstruction of the alimentary tract was performed using the small or large intestine. In such cases, the abdominal procedure was performed by conventional laparotomy. When alimentary reconstruction through the posterior mediastinal route was impossible, the ante-sternal route was adopted. In those cases, the original esophageal hiatus of the diaphragm was closed with suturing.

\section{Repair surgery}

When digestive organ, except for graft conduit, herniating 
Table 1 Patients with postoperative EHH

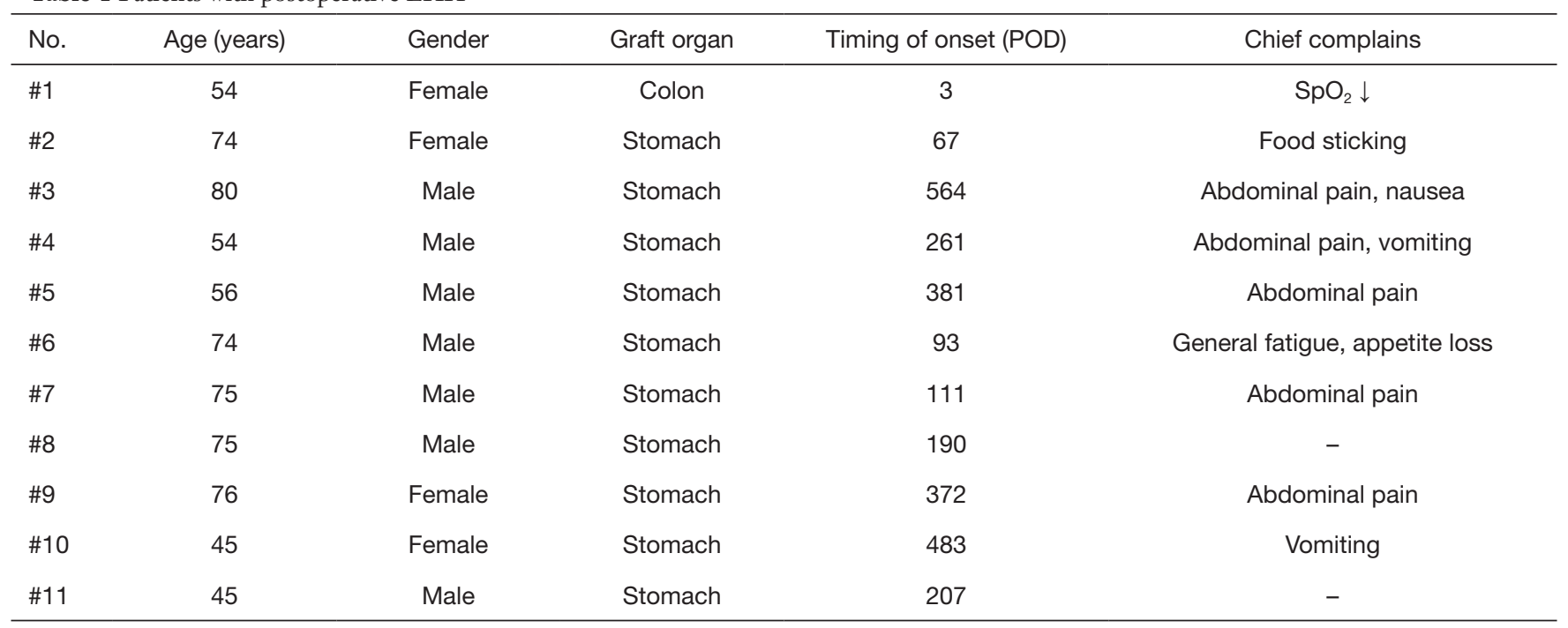

$\mathrm{EHH}$, esophageal hiatal hernia; POD, postoperative days.

into thoracic cavity was detected with chest X-ray and/ or CT examinations, we diagnosed it as postoperative $\mathrm{HH}$ and performed laparoscopic repair surgery for all patients, regardless of symptoms. After pulling-down the herniated organ into abdominal cavity carefully, enlarged esophageal hiatus was tightly closed and graft organ was fixed at diaphragm again using non-absorbable sutures. Moreover, we added colopexy on diaphragm to prevent $\mathrm{HH}$ recurrence.

\section{Statistical analyses}

Descriptive statistics were used to characterize various clinical factors. We used the Mann-Whitney $U$ test to compare the averages of continuous variables and the chi-squared test to compare the discrete variables. The threshold for significance was $\mathrm{P}<0.05$. We used the $\mathrm{JMP}^{\circledR}$ Pro software program, ver. 12.2.0 (SAS Institute, Cary, NC, USA) for these analyses.

\section{Results}

\section{Patients with postoperative $\mathrm{HH}$}

The mean observation period was 34 months (range, 186 months), and scheduled examinations were performed in most cases (84\%). During the study period, 25 patients died of recurrent diseases, and 9 died of other causes. These patients were censored for the occurrence of postoperative
$\mathrm{HH}$ in this study. Eleven (9.7\%) of the 113 patients enrolled in this study were diagnosed with postoperative $\mathrm{HH}$ (7 men and 4 women, Table 1 ).

The chief complaints and symptoms of postoperative $\mathrm{HH}$ varied among the patients. Nine patients experienced some symptoms, such as abdominal pain, while two with no complaints were diagnosed with postoperative $\mathrm{HH}$ by regular follow-up CT. All patients who developed $\mathrm{HH}$ after esophagectomy needed emergent $(n=6)$ or elective surgeries $(\mathrm{n}=5)$ to repair it. The cumulative incidence of $\mathrm{HH}$ is shown in Figure 1. Most cases of postoperative $\mathrm{HH}$ occurred within 1 year, and none occurred 2 years after surgery. Repair surgery was performed an average of 215 days after esophagectomy (range, 3-564 days). There was no marked difference in the occurrence of postoperative $\mathrm{HH}$ between the initial and last half-periods of this study $(7.0 \% \mathrm{vs}$. $12.5 \%, \mathrm{P}=0.32$ ). One patient had repeated herniorrhaphy due to recurrence of $\mathrm{HH}$ at 95 days after surgery to repair the initial HH, 207 days following MIE. He was a carpenter and returned to work, where he worked hard, immediately after both the esophagectomy and the first $\mathrm{HH}$ repair surgery.

Regarding the general features of postoperative $\mathrm{HH}$, the colon was herniated into the left thorax along leftside of the gastric conduit through the esophageal hiatus (Figure 2). One patient had both colon and jejunum herniation, and three had only jejunum herniation. Among the patients with postoperative $\mathrm{HH}$, the hiatus was enlarged, and the edge of the enlarged hiatus was 


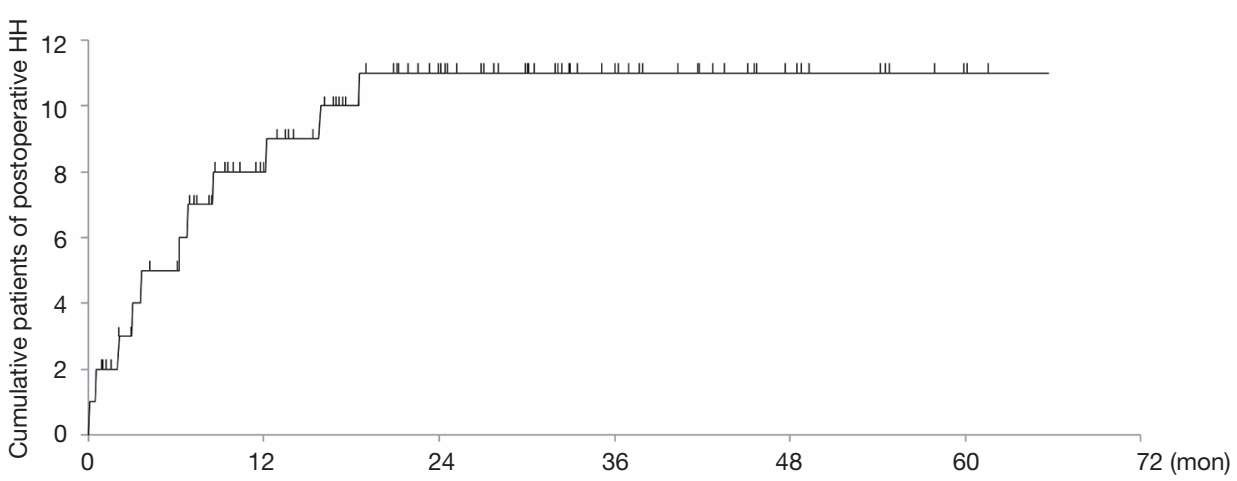

Figure 1 This figure shows the cumulative number of patients with post-operative hiatal hernia $(\mathrm{HH})$ after minimally invasive esophagectomy. The HH occurred within 2 years after the esophagectomy in all cases.

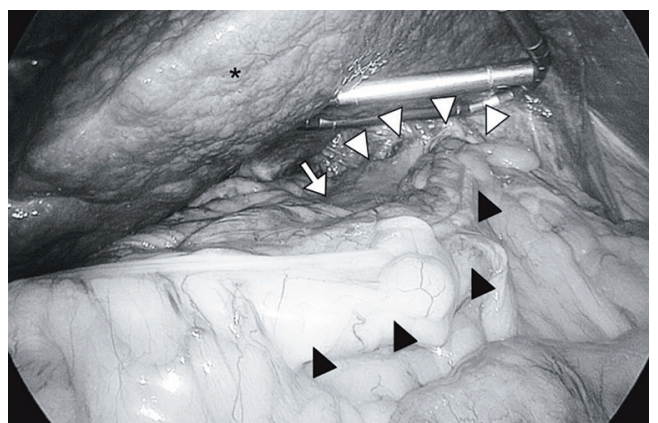

Figure 2 Esophageal hiatus (white arrow head) was enlarged and transverse colon (black arrow head) was herniated into left thorax along with gastric conduit (white arrow). *, lateral segment of liver.

covered by smooth membranous tissues like peritoneum or pleura. Interestingly, the sutures used for the fixation of reconstruction organs at the diaphragm were often observed on the graft or diaphragm, but the knots did not become loosened during repair surgery.

\section{Characteristics of patients undergoing thoracoscopic esophagectomy}

To explore the risk factors of postoperative $\mathrm{HH}$ development, we compared the characteristics of the patients with and without postoperative $\mathrm{HH}$ (Table 2). The ratio of women was higher in the $\mathrm{HH}$ group than in the non-HH group although not to a significant degree $(\mathrm{P}=0.05)$. The preoperative body mass index $(\mathrm{BMI})$ was almost the same between the two groups $(\mathrm{P}=0.99)$. In 21 patients, sliding esophageal $\mathrm{HH}$ was observed before esophagectomy. However, it was not associated with postoperative $\mathrm{HH}$ development.

Regarding the nutritional conditions before esophagectomy, the preoperative albumin level was not markedly different between the two groups, and concomitant illness or smoking habit was not related to postoperative $\mathrm{HH}$ either. All patients with postoperative $\mathrm{HH}$ had clinical stage II or III disease $(\mathrm{P}=0.14)$. Sixty-six patients $(58.4 \%)$ during the study period were administered NAC, and 19 of them (28.8\%) received radiation therapy. Regarding NAC, 10 of 11 patients $(90.9 \%)$ in the $\mathrm{HH}$ group received NAC according to the clinical stage, which was significantly more than in the non-HH group $(\mathrm{P}=0.02)$. Neoadjuvant radiation therapy was not a significant risk factor for postoperative $\mathrm{HH}(\mathrm{P}=0.47)$.

About $70 \%$ of tumor located in middle thoracic esophagus in the patients with esophageal cancer in this series, and there was not significant difference in the aspect of tumor location between $\mathrm{HH}$ and non-HH groups $(\mathrm{P}=0.28)$.

Aside from female gender and the presence of NAC, no significant factors were found to be associated with postoperative $\mathrm{HH}$ development in univariate analyses of the characteristics of the patients in the present study.

\section{Surgical outcomes of thoracoscopic esophagectomy}

To determine whether or not the surgical outcomes might be related to postoperative $\mathrm{HH}$, we compared several surgical factors between the two groups (Table 3). We used a robot to perform the thoracoscopic procedure in 12 patients $(10.6 \%)$ during the study period. However, only one patient with robot-assisted surgery developed postoperative $\mathrm{HH}$. Among the 11 patients in the $\mathrm{HH}$ group, the graft 
Table 2 Clinicopathological feature

\begin{tabular}{|c|c|c|c|c|}
\hline Variable & Total & $\mathrm{HH}$ & Non-HH & $P$ value \\
\hline Age (years) & 66.1 & 66.3 & 64.4 & 0.90 \\
\hline Height (cm) & 162.5 & 161.2 & 162.7 & 0.73 \\
\hline Weight (kg) & 56.5 & 55.6 & 56.6 & 0.90 \\
\hline Preoperative $\mathrm{EHH}$ & 21 & 1 & 20 & 0.39 \\
\hline Smoking & 72 & 7 & 65 & 0.83 \\
\hline \multicolumn{5}{|l|}{ Comorbidity } \\
\hline Heart & 55 & 6 & 49 & 0.68 \\
\hline Albumin (g/dL) & 3.71 & 3.48 & 3.74 & 0.18 \\
\hline Tumor location (Ce/Ut/Mt/Lt/Ae) & $2 / 13 / 65 / 32 / 1$ & $0 / 2 / 4 / 4 / 1$ & $2 / 11 / 61 / 28 / 0$ & 0.28 \\
\hline Clinical stage (0/I/II/III/IV) & $7 / 30 / 33 / 39 / 4$ & $0 / 0 / 5 / 6 / 0$ & $7 / 30 / 28 / 33 / 4$ & 0.14 \\
\hline NAC & 66 & 10 & 56 & $0.02^{*}$ \\
\hline NART & 19 & 1 & 18 & 0.47 \\
\hline
\end{tabular}

*, P<0.05. BMI, body mass index, NAC, neoadjuvant chemotherapy; NART, neoadjuvant radiotherapy; Ce, cervical esophagus; Ut, upper thoracic esophagus; Mt, middle thoracic esophagus; Lt, lower thoracic esophagus; Ae, abdominal esophagus; $\mathrm{HH}$, hiatal hernia; EHH, esophageal hiatal hernia.

organ was the stomach in 10 patients and colon in 1 patient, showing no significance. Ten of 11 patients with postoperative $\mathrm{HH}$ underwent laparoscopic surgery and 1 patient underwent laparotomy for the abdominal procedure. The ratio of laparoscopic surgery was slightly lower in the HH group (91.0\%) than in the non-HH group (93.1\%), although laparoscopic surgery was not statistically related to postoperative $\mathrm{HH}$ development $(\mathrm{P}=0.78)$. The operative time, amount of blood loss, presence of blood transfusion, number of retrieved lymph nodes, timing of leaving bed and length of postoperative hospital stay were not significantly different between the two study groups (Table 3).

The fixation procedure of the conduit organ at the hiatus was historically modulated after noticing the occurrence of postoperative HH. Nine patients $(8.0 \%)$ had no fixation, $36(34.6 \%)$ had fixation by absorbable sutures, and 68 (65.4\%) had fixation by non-absorbable sutures. We initially considered that exact fixation of the graft organ at the esophageal hiatus was crucial for prevention of postoperative $\mathrm{HH}$ development, but it was found to be unlikely to be related $(\mathrm{P}=0.88)$. We suspected that postoperative complications, especially recurrent nerve palsy, aspiration pneumonia or continuous cough, might contribute to postoperative $\mathrm{HH}$ development by drastically increasing the abdominal pressure, but no significant association in these factors was noted between the two groups. Regarding the postoperative nutritional conditions, most patients with esophagectomy had some body weight loss after the surgery. However, the degree of body weight loss showed no association with postoperative $\mathrm{HH}(\mathrm{P}=0.88)$.

To exclude any interaction among the various factors in relation to development of postoperative $\mathrm{HH}$, we should have performed multivariate logistic regression analysis. However, number of patients enrolled in this study was small to analyze the independent risk factor of $\mathrm{HH}$ development.

\section{Discussion}

Previous studies have reported that the incidence of internal hernia, including esophageal HH, is higher in MIS than in conventional procedures (8-10). However, this issue has not 
Table 3 Outcomes of thoracoscopic esophagectomy

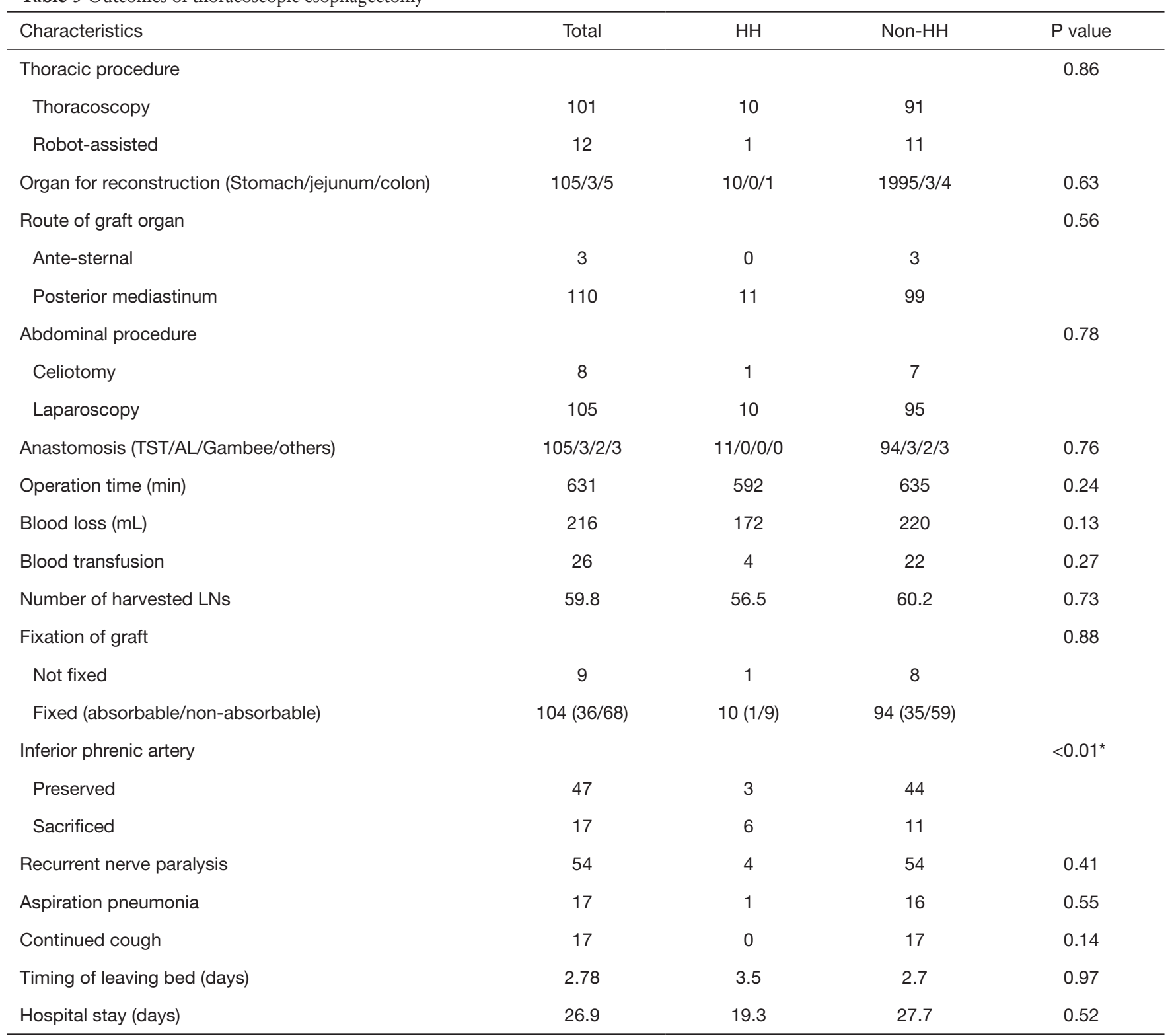

*, $\mathrm{P}<0.05$. TST, triangulating stapling technique, AL, Albert-Lembert suture, LNs, lymph nodes.

been well addressed, as the risk factors of $\mathrm{HH}$ development after MIE have been unclear. The present study was based on the completely performed surveillance of patients with esophagectomy. In addition, all patients were treated by thoracoscopic surgery as part of the thoracic procedure during the study period, possibly showing minimal selection bias. This report showed that the cumulative incidence of postoperative $\mathrm{HH}$ plateaued at about $10 \%$ at 2 years after esophagectomy with 3-stage lymph node dissection for esophageal cancer with MIS, and that the presence of NAC was a risk factor of postoperative HH development.

The timing at which postoperative $\mathrm{HH}$ occurred varied widely, ranging from 3 to 564 days after MIE. As esophageal cancer still has a high mortality and some patients with esophagectomy may develop recurrence before suffering from postoperative $\mathrm{HH}$, the number of patients with postoperative $\mathrm{HH}$ might be underestimated. Previous studies have found that postoperative $\mathrm{HH}$ occurred in $0.8-7.9 \%$ of patients after conventional esophagectomy. Regarding MIE, a few reports have described the incidence 
of $\mathrm{HH}$ as $4.5-26 \%(9,10,15)$. Based on both our present and these previous findings, the incidence of postoperative $\mathrm{HH}$ after MIE seems to be higher than that after conventional esophagectomy. While the reason for this was not addressed in the present study due to its comparative nature, we speculate that the minimal adhesion that occurs after MIS may be responsible (16-18). Furthermore, among the 11 patients with postoperative $\mathrm{HH}$ in our series, most $(n=9)$ complained of abdominal pain resulting from the incarceration or strangulation of the intestine, while $2 \mathrm{did}$ not have any symptoms when $\mathrm{HH}$ was diagnosed (Table 1). These findings support the careful follow-up of patients for at least 2 years after esophagectomy.

We investigated the risk factors for postoperative $\mathrm{HH}$ development from two aspects: based on patient characteristics and based on surgical factors. First, we determined the patient characteristics. Previous reports have suggested that the pressure gap between the chest and abdominal cavity might cause postoperative $\mathrm{HH}$ development $(9,19)$, and several factors may be responsible for increasing this pressure gap. Ganeshan et al. reported that female gender and a low BMI were risk factors of $\mathrm{HH}$ development after esophagectomy (15). Indeed, in the present study, we observed a tendency toward a higher female-to-male ratio in the $\mathrm{HH}$ group, although it was not significant and that reason was not clear. We also encountered an interesting patient with repeated postoperative $\mathrm{HH}$ who was a carpenter by trade. $\mathrm{He}$ returned to his full work load immediately after both esophagectomy and the first hernia repair, which drastically increased his abdominal pressure, potentially leading to $\mathrm{HH}$ recurrence. However, the BMI was not associated with postoperative $\mathrm{HH}$ in our series. We therefore consider that the pressure gap between the chest and abdominal cavity alone does not account for postoperative $\mathrm{HH}$ development. Some concomitant disease might contribute to $\mathrm{HH}$ development, but our univariate analyses did not suggest that any particular disease was a significant risk factor in HH development after MIE.

We focused on NAC in the present study. More than $90 \%$ of esophageal cancer is squamous cell carcinoma in Japan (20), and chemotherapy and radiotherapy have proven to be effective for treating esophageal cancer $(4,21)$. NAC has been shown to extend the overall survival of patients with esophageal cancer and is recommended for patients with lymph node metastases at the diagnosis of esophageal cancer (4). According to the Japanese Guidelines for Diagnosis and Treatment of Carcinoma of the Esophagus, edited by the Japanese Esophageal Society, we generally administer NAC for patients with cStage II and III esophageal cancer, except for the patients without agreement for NAC. However, NAC is known to cause a high incidence of postoperative complications. Benjamin et al. suggested that NAC might be a risk factor of postoperative $\mathrm{HH}$ development after laparoscopic transhiatal esophagectomy, although no statistical significance was observed (19). Gooszen et al. also have reported that chemoradiotherapy might be one of risk factor of $\mathrm{HH}$ after esophagectomy, including both open and MIE (22). However, in the present study, we clearly showed that NAC was a risk factor of $\mathrm{HH}$ development after thoracoscopic esophagectomy. NAC induces fragility of tissues and delays wound healing, which might cause postoperative $\mathrm{HH}$ development. In addition, the synergistic effect of reducing tissue adhesion by MIS might play an important role $(8-10,16,17)$. Indeed, we often observed during repair surgery for HH that the sutures remained on the graft or diaphragm while the knots were maintained, suggesting that tearing of either tissue could happen with exposure to sufficient external pressure. In all cases, postoperative $\mathrm{HH}$ occurred within 2 years after surgery. Thus, perioperative fragility of tissues might be an important factor influencing postoperative $\mathrm{HH}$ development. In recent decades, NAC has been introduced and applied in patients with cStage II or III tumors. Therefore, the incidence of postoperative $\mathrm{HH}$ after esophagectomy might be lower in previous reports than in the current study. cStage was not a risk factor of $\mathrm{HH}$ development, although NAC was. It might be because some patients underwent surgery without NAC in our series.

We frequently encountered an enlarged hiatus in repair surgery. Hence, by reviewing videos, we retrospectively checked whether or not right inferior phrenic artery was preserved at abdominal procedure, because atrophic changes in diaphragm might be due to the sacrifice of the left inferior phrenic artery. Unfortunately, we could check only 64 cases. Nevertheless, the ratio of artery preservation was significantly lower in the HH group than in the non$\mathrm{HH}$ group and the preservation of the left inferior phrenic artery significantly contributed to postoperative $\mathrm{HH}$ development $(\mathrm{P}<0.01$, Table 2).

The fixation of the graft conduit to the diaphragm is considered to be the most important method of surgical prophylaxis for preventing post-operative $\mathrm{HH}$ development. Nine patients had no fixation, and 104 had fixation using absorbable or non-absorbable sutures; 
however, the fixation of the graft at the diaphragm was not found to be a significant factor influencing postoperative HH development. Nevertheless, we believe that the fixation of the graft organ at the diaphragm might be helpful for straightening the graft in order to prevent the bending of the redundant conduit in the mediastinum after the operation as well as to prevent postoperative $\mathrm{HH}$ development. In most patients, colon was herniated into the thorax. Therefore, colopexy on diaphragm is one of recommended methods to prevent post-operative $\mathrm{HH}$ development (23), especially for the HH high-risk patients, and now we initiatively add the procedure for the patients with NAC.

Several limitations associated with the present study warrant mention, including its retrospective nature and the diverse surgeries performed as well as the backgrounds of the patients themselves. However, postoperative $\mathrm{HH}$ is a common complication of thoracoscopic esophagectomy, and the present study is an important one for investigating the risk factors for postoperative $\mathrm{HH}$. We should take additional precautions in order to prevent the development of postoperative $\mathrm{HH}$ in patients who undergo MIE after NAC.

In the current study, we showed a relatively high incidence of postoperative $\mathrm{HH}$ after MIE. Analyses for the risk factors of $\mathrm{HH}$ development revealed that NAC was a major risk factor.

\section{Acknowledgments}

The authors are grateful to Dr. Osamu Ikeda, Saga-Ken Medical Center Koseikan, for suggesting the topic treated in this paper.

Funding: None.

\section{Footnote}

Reporting Checklist: The authors have completed the STROBE reporting checklist. Available at http://dx. doi. org/10. 21037/jtd-20-1335

Data Sharing Statement: Available at http://dx.doi. org/10.21037/jtd-20-1335

Peer Review File: Available at http://dx.doi.org/10.21037/jtd20-1335

Conflicts of Interest: All authors have completed the ICMJE uniform disclosure form (available at http://dx. doi. org/10.21037/jtd-20-1335). The authors have no conflicts of interest to declare.

Ethical Statement: The authors are accountable for all aspects of the work in ensuring that questions related to the accuracy or integrity of any part of the work are appropriately investigated and resolved. The study was conducted in accordance with the Declaration of Helsinki (as revised in 2013). The study was approved by Institutional Review Board of Saga University Hospital (ID; 2017-04-22) and informed consent was taken from all the patients.

Open Access Statement: This is an Open Access article distributed in accordance with the Creative Commons Attribution-NonCommercial-NoDerivs 4.0 International License (CC BY-NC-ND 4.0), which permits the noncommercial replication and distribution of the article with the strict proviso that no changes or edits are made and the original work is properly cited (including links to both the formal publication through the relevant DOI and the license). See: https://creativecommons.org/licenses/by-nc-nd/4.0/.

\section{References}

1. Pennathur A, Gibson MK, Jobe BA, et al. Oesophageal carcinoma. Lancet 2013;381:400-12.

2. Jemal A, Bray F, Center MM, et al. Global cancer statistics. CA Cancer J Clin 2011;61:69-90.

3. Taylor MD, LaPar DJ, Davis JP, et al. Induction chemoradiotherapy and surgery for esophageal cancer: survival benefit with downstaging. Ann Thorac Surg 2013;96:225-30.

4. Ando $\mathrm{N}$, Kato $\mathrm{H}$, Igaki $\mathrm{H}$, et al. A randomized trial comparing postoperative adjuvant chemotherapy with cisplatin and 5-fluorouracil versus preoperative chemotherapy for localized advanced squamous cell carcinoma of the thoracic esophagus (JCOG9907). Ann Surg Oncol 2012;19:68-74.

5. Tachimori Y, Ozawa S, Numasaki H, et al. Comprehensive Registry of Esophageal Cancer in Japan, 2010. Esophagus 2017;14:189-214.

6. Cuschieri A, Shimi S, Banting S. Endoscopic oesophagectomy through a right thoracoscopic approach. J R Coll Surg Edinb 1992;37:7-11.

7. Luketich JD, Alvelo-Rivera M, Buenaventura PO, et al. Minimally invasive esophagectomy: outcomes in 222 patients. Ann Surg 2003;238:486-94. 
8. Nguyen NT, Roberts P, Follette DM, et al. Thoracoscopic and laparoscopic esophagectomy for benign and malignant disease: lessons learned from 46 consecutive procedures. J Am Coll Surg 2003;197:902-13.

9. Willer BL, Worrell SG, Fitzgibbons RJ Jr, et al. Incidence of diaphragmatic hernias following minimally invasive versus open transthoracic Ivor Lewis McKeown esophagectomy. Hernia 2012;16:185-90.

10. Kent MS, Luketich JD, Tsai W, et al. Revisional surgery after esophagectomy: an analysis of 43 patients. Ann Thorac Surg 2008;86:975-83.

11. Kuwano H, Nishimura Y, Oyama T, et al. Guidelines for Diagnosis and Treatment of Carcinoma of the Esophagus April 2012 edited by the Japan Esophageal Society. Esophagus 2015;12:1-30.

12. Noshiro H, Yoda Y, Hiraki M, et al. Survival outcomes of 220 consecutive patients with three-staged thoracoscopic esophagectomy. Dis Esophagus 2016;29:1090-9.

13. Noshiro H, Urata M, Ikeda O, et al. Triangulating stapling technique for esophagogastrostomy after minimally invasive esophagectomy. Surgery 2013;154:604-10.

14. Noshiro H, Iwasaki H, Kobayashi K, et al. Lymphadenectomy along the left recurrent laryngeal nerve by a minimally invasive esophagectomy in the prone position for thoracic esophageal cancer. Surg Endosc 2010;24:2965-73.

15. Ganeshan DM, Correa AM, Bhosale P, et al. Diaphragmatic hernia after esophagectomy in 440 patients with long-term follow-up. Ann Thorac Surg

Cite this article as: Iwasaki H, Tanaka T, Miyake S, Yoda Y, Noshiro H. Postoperative hiatal hernia after minimally invasive esophagectomy for esophageal cancer. J Thorac Dis 2020;12(9):4661-4669. doi: 10.21037/jtd-20-1335
2013;96:1138-45.

16. Vallböhmer D, Hölscher AH, Herbold T, et al. Diaphragmatic hernia after conventional or laparoscopicassisted transthoracic esophagectomy. Ann Thorac Surg 2007;84:1847-52.

17. Schippers E, Tittel A, Ottinger A, et al. Laparoscopy versus laparotomy: comparison of adhesion-formation after bowel resection in a canine model. Dig Surg 1998;15:145-7.

18. Aly A, Watson DI. Diaphragmatic hernia after minimally invasive esophagectomy. Dis Esophagus 2004;17:183-6.

19. Benjamin G, Ashfaq A, Chang YH, et al. Diaphragmatic hernia post-minimally invasive esophagectomy: a discussion and review of literature. Hernia 2015;19:635-43.

20. Tachimori Y, Ozawa S, Numasaki H, et al. Comprehensive Registry of Esophageal Cancer in Japan, 2009. Esophagus 2016;13:110-37.

21. Kohei Shitara, Kei Muro. Chemoradiotherapy for Treatment of Esophageal Cancer in Japan: Current Status and Perspectives. Gastrointest Cancer Res 2009;3:66-72.

22. Gooszen JAH, Slaman AE, van Dieren S, et al. Incidence and Treatment of Symptomatic Diaphragmatic Hernia After Esophagectomy for Cancer. Ann Thorac Surg 2018;106:199-206.

23. Argenti F, Luhmann A, Dolan R, et al. Diaphragmatic hernia following oesophagectomy for oesophageal cancer Are we too radical? Ann Med Surg (Lond) 2016;6:30-5. 